\title{
Sistematización Educación infantil e infancias. Análisis documental del Programa de Educación Preescolar de la Escuela de Educación de la Fundación Universitaria Monserrate - Unimonserrate ${ }^{1}$
}

\author{
Systematization of early childhoods. Documentary analysis of the preschool \\ education program of the Education School of Fundación Universitaria \\ Monserrate - Unimonserrate
}

Diana Milena Trujillo Mahecha ${ }^{2}$

\begin{abstract}
Resumen
La escuela de educación de la Fundación Universitaria Unimonserrate, como agente formativo de maestros y maestras, ha generado diversos espacios de reflexión y formación que buscan asumir un rol crítico frente a esta labor. Este trabajo de sistematización tuvo como objetivo recuperar, organizar, analizar y reflexionar las producciones escritas del programa de licenciatura en educación preescolar, para identificar las construcciones que se han adelantado entorno a las infancias y a la educación infantil en la formación de docentes. Se respondió a la necesidad de dar cuenta de las dinámicas y experiencias pedagógicas que se han estado construyendo durante los últimos cuatro años en el programa. Partiendo de la posibilidad heurística que tiene la experiencia como motivadora de nuevas preguntas y logros cognoscitivos que aportan conocimiento científico para la construcción de nuevos saberes. Identificar los modos de hacer, las mediaciones del maestro, las concepciones de infancia y de desarrollo infantil, posibilitó visibilizar la realidad de la acción educativa en este ciclo y proponer otros sentidos a las prácticas y a los escenarios pedagógicos en los que las infancias, transitan, habitan y viven.
\end{abstract}

Palabras claves: Educación infantil; Infancias; Sistematización.

\section{Abstract}

The school of education of the Fundación Universitaria Monserrate - Unimonserrate, as a training agent for teachers, has generated various spaces for reflection and training that seek to assume a critical role in this work. This systematization work aimed to recover, organize, analyze and reflect on the written productions of the preschool education degree program, to identify the constructions that have been carried out around childhood and early childhood education in teacher training. We responded to the need to account for the dynamics and pedagogical experiences that have been building during the last four years in the program. Starting from the heuristic possibility that experience has as a motivator of new questions and cognitive achievements that provide scientific knowledge for the construction of new knowledge. Identify the ways of doing, the mediations of the teacher, the conceptions of childhood and childhood development, made it possible to visualize the reality of the educational action in this cycle and propose other meanings to the practices and the pedagogical scenarios in which the childhood, transit, they live and live.

Keywords: Infant education; Childhood; Systematization.

\footnotetext{
${ }^{1}$ Artículo de reflexión.

${ }^{2}$ Mg. Educación. Docente Escuela de Educación Uniminuto. https://orcid.org/0000-0003-2478-579X

E-mail: diana_milena.trujillo@hotmail.com

${ }^{*}$ Cómo citar este artículo: Trujillo, D. (2018). Sistematización Educación infantil e infancias. Análisis documental del Programa de Educación Preescolar de la Escuela de Educación de la Fundación Universitaria Monserrate - Unimonserrate. Hojas y Hablas, (16), 75-83. DOI: 10.29151/hojasyhablas.n16a5
} 


\section{Introducción}

La sistematización de experiencias nace en América Latina a fines de los años setenta, y fue pensada como forma de abordaje y reconstrucción de experiencias de educación popular para construir conocimiento en conjunto con los sujetos intervinientes, es "una forma específica de investigación que permite la recuperación, a posteriori de la práctica, de los saberes y los conocimientos que han sido eficaces para operar sobre la realidad". (Iovanovich, 2007). En la actualidad, esta metodología de trabajo es una herramienta de la investigación cualitativa con mayor impacto en los ámbitos sociales.

El objetivo principal de este proceso de sistematización fue reconstruir y racionalizar desde cuatro dimensiones: ontológica abordando los ejes transversales educativos de ser y convivir, metodológica haciendo un análisis de las herramientas, técnicas e instrumentos vinculados a las propuestas pedagógicas, epistemológica ubicando la producción de conocimiento que emerge de la experiencia desde la construcción crítica de nuevas categorías de análisis y política problematizando para qué y para quién se sistematiza la experiencia. (García y Morgan, 2007)

La muestra documental de esta sistematización está conformada por 33 documentos, entre los cuales se encuentran apuestas metodológicas para diplomados con sus procesos de evaluación, productos como presentaciones y paneles, estudios y proyectos de investigación del programa de educación preescolar. En este proceso se organizó en categorías la información encontrada, con un enfoque descriptivo, analítico y proyectivo, desde la reflexión, la crítica constructiva y un sentido altamente valorativo para identificar los principales aprendizajes generados, transformando el conocimiento tácito en conocimiento explícito que puede ser generalizado y puesto a disposición de otras realidades.

Esto permitió la construcción de conocimiento para la escuela y a su vez la posibilidad de retomar las voces valiosas de los diferentes actores, evitando que se pierda información valiosa para proponer planes de acción y una sublínea de investigación para la escuela de educación.

Figura 1. Corpus documental.

\section{Documentación revisada}

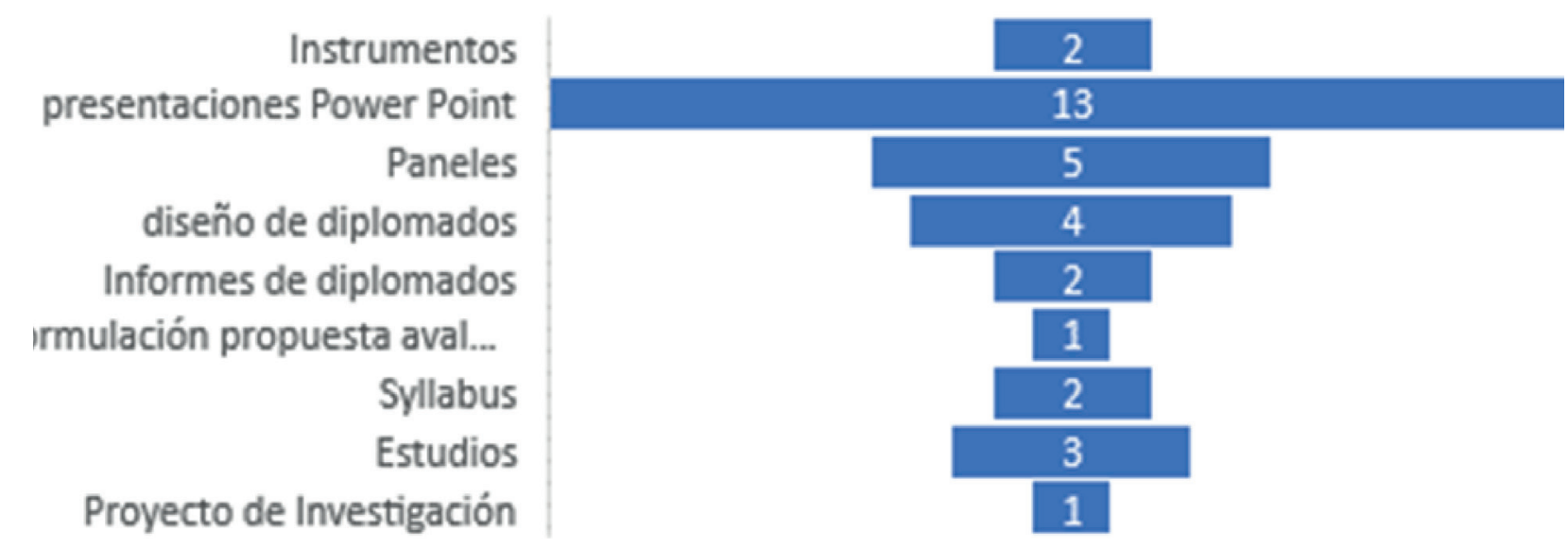

Fuente: elaboración propia. 
La organización del corpus documental a sistematizar se llevó a cabo en una matriz de conceptos. Para construirla, las siguientes preguntas orientaron el proceso: ¿cuál es el sentido del trabajo pedagógico con los niños y niñas de 0 a 3 años?, ¿Cuál es el rol del maestro en los momentos de desarrollo de la educación infantil?; ¿cuáles son los propósitos fundamentales en la educación inicial?; ¿cuáles son los componentes de la acción pedagógica en la educación infantil?; ¿Cuáles son las apuestas metodológicas? y ¿cuáles son los enfoques que inspiran el trabajo pedagógico? En este ejercicio emergen las categorías de análisis: concepción de niño, niña y maestro, el sentido de la educación inicial y la escuela como institución; visibilizando los retos y la problematización de lo encontrado y develando los hallazgos en cada uno de los documentos.

En este proceso se encuentra que las producciones escritas de los maestros de la Fundación Universitaria Unimonserrate, de la licenciatura en educación preescolar están cargados de significaciones, de historia, de formas de ver la vida, de conceptos logrados colectiva e individualmente. Estos discursos se han ido entretejiendo, conformando, adrede o no, como un discurso institucional. El énfasis en el diseño de ambientes, en el reconocimiento social y cultural de los niños y las niñas, de la multiplicidad de infancia (porque una infancia no lograría abarcar la multiplicidad de factores que la conforman) hace parte del entramado discursivo de la escuela de educación.

Este análisis documental fue un proceso alejado de toda objetividad, que fue develando similitudes, diferencias y tensiones en varios conceptos relacionados con la infancia y la educación infantil. De la sistematización emergen nuevas categorías que permiten dan respuesta a las preguntas orientadoras iniciales y que dialogan con los hallazgos. Una concepción de las infancias como una construcción social; prácticas pedagógicas que van más allá de la pre-escolaridad; un maestro que reconoce y reconfigura las concepciones de infancia desde la diversidad que generara espacios y condiciones diversas para el desarrollo integral de los niños, niñas, sus familias y comunidades, y la episteme del programa como una construcción social académica.

\section{Concepción de infancias: La infancia como con- strucción social}

Sin duda, el punto en común de todos los documentos es la concepción de infancia como una construcción social. Los maestros han venido tejiendo su discurso en torno a las tensiones mundiales del reconocimiento de la infancia y de las infancias, y han atribuido características a estas nuevas y diversas maneras de verla, de ninguna forma se reducen a la de un sujeto pasivo que asimila el mundo que lo rodea, sino que, por el contrario

Esta infancia que actúa participa, posee saberes y formas particulares de reconocer y descubrir el mundo y múltiples lenguajes para expresarse. En esa medida la infancia es mirada como potente, no carente, un momento del ciclo vital, determinante, en el que todas las posibilidades están dadas para potenciar el desarrollo. Una infancia que se desarrolla a diferentes ritmos y momentos. (Espitia, 2017 p. 12).

En este sentido, la licenciatura en educación preescolar de la Escuela de Educación ha venido determinando que la infancia es plural porque no es única, ni homogénea, porque es social y universal; no solo se trata de la edad o de la biología, sino que las infancias están determinadas histórica, cultural y políticamente. Esto lleva a que la educación infantil también se empiece a entender de formas plurales. La infancia ha venido construyéndose entonces como ciudadana, con posibilidades para desarrollar procesos de participación, según su edad y circunstancias, que va construyendo su identidad y cultura en los encuentros con las costumbres, prácticas, ideas, pensamientos y formas de interacción con su entorno. Una infancia que se desarrolla a diferentes ritmos y momentos, que 
se entiende desde la complejidad en su abordaje, comprendiendo su multidimensionalidad y globalidad, como sujetos activos, con capacidad para responder, proponer y decidir. Esta concepción de infancia ha traído consigo la atribución de características particulares al niño y a la niña, que no solo se relaciona con un agente educativo sino como un Sujeto de derechos que configura diferentes formas de ver el mundo según su contexto y diversidad. En ese sentido, los niños y las niñas no sólo interiorizan la sociedad y la cultura, sino que también contribuyen a la reproducción, construcción y al cambio cultural de un modo activo, al tratar cotidianamente con la cultura procedente de los adultos, debido a la estructura social existente, y con aquella que producen simultáneamente con sus pares.

\section{Pedagógica - Educación - Prácticas: la nueva concepción frente a las infancias implica nuevas prácticas pedagógicas más allá de la pre-esco- laridad}

Los debates académicos y políticos de la última década son retomados en los textos de los maestros, como un soporte para dar sentido a la educación inicial, muestra de esto se encuentra en el syllabus de la maestra Consuelo Martín,

(...) un derecho impostergable de la primera infancia que propende por garantizar el desarrollo armónico e integral de los niños y niñas y busca reducir la segregación social, constituyéndose así, en una herramienta que brinda orientaciones a los jardines infantiles $\mathrm{y}$ colegios que atienden niños y niñas en primera infancia para reflexionar y estructurar su práctica pedagógica acorde con las características y particularidades de las instituciones, los niños y niñas, y sus familias.

Preguntas como ¿para qué van los niños y niñas a los espacios de educación inicial?; ¿cuál es el sentido de la educación inicial? Están presentes en las diversas apuestas formativas de la Universidad. La educación inicial se convierte en un derrotero de la atención integral que da a la educación características como la intención, el sentido, el desarrollo, el reconocimiento del contexto y de la particularidad de las infancias. Implica la generación de ambientes enriquecidos $y$ de experiencias que favorezcan las interacciones de las niñas y niños con el ambiente, con el maestro y entre otras múltiples relaciones. Esta visión de la infancia implica una mirada amplia de la misma, lo que supone un estudio interdisciplinar, en el que confluyen diferentes perspectivas: psicológicas, filosóficas, antropológicas, que complementan la mirada pedagógica. A partir de las particularidades sociales, culturales, históricas y políticas de la infancia y de sus potencialidades de donde emerge el acto educativo. Actos reflexionados para la transformación e innovación. La educación infantil implica el reconocimiento de la multidimensionalidad, complejidad e interdisiciplinariedad de las acciones con la infancia. La educación infantil se define desde el conjunto de prácticas, modelos, metodologías que le apuestan al desarrollo integral, crecimiento y cuidado de los niños y niñas de 0 a 6 años, en el marco de los contextos sociales, políticos, económicos, culturales, geográficos, ambientales e históricos que los constituyen, tal y como lo define la política pública de estado para el desarrollo integral de la primera infancia de Cero a Siempre,

La educación inicial es un derecho de los niños y las niñas menores de seis (6) años de edad. Se concibe como un proceso educativo y pedagógico intencional, permanente y estructurado, a través del cual los niños y las niñas desarrollan su potencial, capacidades y habilidades en el juego, el arte, la literatura y la exploración del medio, contando con la familia como actor central de dicho proceso. (Ley 1804, 2016).

La especialidad de esta educación encierra las relaciones que se tejen entre el cuidado y la pedagogía en pro de la calidad de vida de la infancia. Por ende, hay grandes retos para reconocer nuevas prácticas pedagógicas. Nuevos sentidos 
de las praxis, imaginarios de los y las maestras. El acto educativo es una atmosfera que permite construir conocimiento.

Las investigaciones, las nuevas tensiones y elaboraciones en la pedagogía y la didáctica de las últimas décadas recrean un nuevo panorama del trabajo con las infancias desde una comprensión del desarrollo integral que no sucede de manera lineal, secuencial, acumulativa, siempre ascendente, homogénea, prescriptiva e idéntica para todos los niños y las niñas, sino que se expresa de manera particular en cada uno. La interacción con una amplia variedad de actores, contextos y condiciones es significativa para el potenciamiento de las capacidades y de la autonomía progresiva. El desarrollo ocurre a lo largo de todo el ciclo vital y requiere de acciones que lo promuevan más allá de la primera la infancia. De aquí la necesidad de pasar de la pre -escolaridad al reconocimiento de nuevas prácticas y modalidades para el trabajo con las infancias. De este modo la educación inicial se convierte en un derecho de la infancia que propende por garantizar el desarrollo armónico e integral de los niños y niñas y busca generar equidad e igualdad en el desarrollo social, generando, a través de experiencia la transformación de vida de todos los niños, niñas y sus familias. El desarrollo infantil entendido como un proceso en el que existen múltiples caminos y se caracteriza por un funcionamiento irregular de avances y retrocesos, que toma distancia de la postura frente a que la educación inicial centre su trabajo en el desarrollo de competencias, entendidas como el hacer, saber hacer y poder hacer.

El rol del maestro como un ser humano que reconoce y reconfigura las concepciones de infancia desde la diversidad y así mismo es capaz de generar espacios y condiciones diversas para el desarrollo integral de los niños, niñas, sus familias y comunidades. El maestro en educación Infantil tiene el reto de acompañar al bebé, al niño, la niña y sus familias en la construcción de los significados culturales que portan los objetos, las acciones, los gestos, los modos de actuar, los festejos, las costumbres, etc. Supone también ofrecer experiencias de observación, exploración y experimentación para ampliar y enriquecer el conocimiento de las características del mundo físico, social y natural. Enseñar en la educación inicial es dar: conocimiento, afecto, confianza, calidez, ternura y cuidado; es acunar desde los primeros años con brazos firmes pero abiertos que ofrezcan seguridad y posibilidad de autonomía; es alertar sobre los peligros, es mostrar el mundo y cómo andar en él, es saber retirarse cuando el bebé, el niño y la niña manifiestan que pueden resolver la situación por sí solos.

El programa de licenciatura en educación preescolar tiene una postura epistemológica que se ubica en la producción de conocimiento. En la revisión de los documentos se encuentra que este conocimiento emerge del dialogo entre los desarrollos teóricos y la experiencia, esto da cuenta de un proceso continuo de reflexión entre la teoría y la práctica. La política pública también cobra fuerza como un eje del trabajo pedagógico. Las actividades rectoras: literatura, juego, arte y exploración del medio se hacen presentes en cada uno de los documentos. En literatura se cita a Cabrejo, Bonnafé, Yolanda Reyes, y M. E. López; se hace una referencia especial al trabajo con los libros álbum, las nanas, arrullos y otras manifestaciones de la tradición oral. El programa hace énfasis en la pedagogía crítica para el despliegue de una praxis propia de los actores de la modalidad, potenciadora del desarrollo integral de los niños y las niñas en la primera infancia. La documentación pedagógica cumple un papel importante en este proceso de reflexión entre la teoría y la práctica. Se evidencia una postura política fundamentada en la atención integral para la primera infancia. La Fundación Universitaria Unimonserrate ha estado repensando su programa de licenciatura en Educación Preescolar con miras a dar respuesta a los avances y demandas que se han venido posicionando en materia de Educación Infantil en el país, y que aportan en la definición del 
perfil de los y las egresadas del programa y de la organización misma de este que, aunque siga denominándose preescolar ya ha tenido importantes reestructuraciones que responden al sentido mismo de la Educación Infantil. El programa asume una metodología en la que le permite a sus estudiantes ser protagonistas de su propio proceso, con un carácter teórico-práctico, que se concreta en el análisis, reflexión y resignificación de las experiencias y relaciones que se establecen en la primera infancia. Las estrategias pedagógicas en el proceso de formación de los estudiantes son sesiones presenciales de formación, trabajo autónomo y socialización desde la perspectiva de diálogo de experiencias. Se proponen escenarios posibles de indagación o exploración, que configuran la complejidad de la producción de discursos y prácticas pedagógicas en educación inicial hoy: académico, práctico y político.

Los estudios adelantados en el programa de carácter exploratorio, en donde el objetivo es profundizar en los elementos que se deben tener en cuenta para la formación de maestras en torno a la Educación Infantil de niños y niñas entre 0 y 3 años, buscan encontrar pistas que permitan fortalecer el programa en su etapa de reestructuración, así como algunas líneas de investigación relacionadas con este campo en particular. La investigación se sustenta en el enfoque de investigación-acción dado que se centra en la propia práctica y busca su re- significación desde la reflexión en y sobre la acción, apoyado en la incorporación del modelo de formación en documentación pedagógica de la escuela de Educación. El programa se enfrenta a unos retos desde unas apuestas metodológicas: a la formación de maestras y maestros de primera infancia, conscientes de la responsabilidad que implica el trabajo sobre todo con los niños y niñas entre 0 y 3 años, pues aunque las políticas públicas han avanzado en materia de Educación Infantil y contamos hoy con un lineamiento curricular muy orientador, aún hay un gran vacío en lo que tiene que ver con los niños y niñas de 0 a 3 años, es decir, sigue habiendo poca literatura que ori- ente el trabajo pedagógico con los más pequeños, y una insuficiente sistematización del saber construido desde la práctica. Razón por la cual desde la Escuela de Educación de la Unimonserrate se pretende contar con algunos horizontes en torno a este tema que posibiliten continuar asumiendo con responsabilidad la formación de maestros y maestras de Educación Infantil.

Rol del maestro: un maestro que reconoce y reconfigura las concepciones de infancia desde la diversidad y así mismo es capaz de generar espacios y condiciones diversas para el desarrollo integral de los niños, niñas, sus familias y comunidades.

Por supuesto el reconocimiento social de la infancia y de la educación inicial, trae consigo la reconfiguración del rol del maestro. El rol es marcado en la Escuela de Educación por varios aspectos, dentro de los que se encuentran dos relevantes:

Primero, la multiplicidad de infancias implica la generación de multiplicidad de espacios y por ende el maestro debe reconfigurar su función tradicional de la enseñanza, para asumir diferentes formas de hacer y ser en el contexto de la educación infantil. Se busca romper el paradigma del maestro que enseña, al maestro que construye, transforma y crea; pero también aprende en su relación y en las interacciones con los niños, las niñas y sus familias.

Segundo, las funciones y roles del maestro también están determinadas en la escuela por la importancia que se le da al diseño de ambientes como estrategia central que facilita la relaciones con los niños, niñas y la construcción colectiva de los procesos de aprendizaje. En este sentido el maestro tiene funciones tales como las expresadas en algunos documentos relacionadas con diseñar, estar atento, interactuar, organizar la oferta lúdica, brindar seguridad, observar y escuchar sensiblemente.

La escuela de Educación busca que sus 
maestros adquieran habilidades concretas para la práctica educativa, para la investigación y del componente disciplinar, una fundamentación en su estructura de pensamiento y del ámbito comunicativo básico (interpretación, divulgación, argumentación). Dicha plataforma se adquiere en la medida en que exista un nivel de sensibilización política, cultural, social, científica, etc., en otras palabras, cuando la persona decide por su propia cuenta formarse y ampliar su matriz cultural y su forma de relación con el mundo.

El rol docente está marcado por una tendencia a considerarlo como un agente mediador entre los niños, las niñas y el mundo, sin desconocer que el maestro en sí tiene saberes, experiencias e intereses que deben ser retomados en las acciones pedagógicas.

El trabajo del docente de educación inicial se ve como el de un artesano, un trabajo dedicado, complejo, atento a la trascendencia social de su labor. Que no es un mero técnico aplicador de currículos, sino que reflexiona sobre su propia práctica documentándola y poniéndola en discusión con otros. Un profesional comprometido con la realidad social y cultural, con capacidades para gestionar proyectos educativos innovadores. (Martín, 2018).

Entre otros aspectos relevantes, se encuentran en los documentos: un docente, que escucha en un sentido amplio, observador, sensible, dispuesto emocional y corporalmente a acoger a los niños y niñas; el maestro debe reconocerse y potenciar de forma intencionada; existe un cambio de las relaciones, horizontal, bidimensional, de construcción colectiva.

El maestro en educación Infantil tiene el reto de acompañar al bebé, al niño, la niña y sus familias en la construcción de los significados culturales que portan los objetos, las acciones, los gestos, los modos de actuar, los festejos, las costumbres, etc.
Supone también ofrecer experiencias de observación, exploración y experimentación para ampliar y enriquecer el conocimiento de las características del mundo físico, social y natural.

\section{La episteme del Programa como una construc- ción social académica}

En los documentos se encuentra que para comprender el desarrollo infantil se citan a autores como Vygotsky, Bruner, Malaguzzi y Hoyuelos, y se hace énfasis en un enfoque socio - cultural, que ratifica las enormes potencialidades y posibilidades de aprendizaje y desarrollo que tienen los niños y niñas de primera infancia.

El enfoque socio-cultural, en el ámbito de lo académico, especialmente con los aportes de Bruner, para comprender las interacciones y fortalecer el sistema de apoyo adulto-bebés. Así como, desde lo práctico, el trabajo con los relatos de vida, que se propone como estrategia de intervención con padres y cuidadores. (Martín y Durán, 2016 p. 21)

Este enfoque socio-critico es una apuesta investigativa que se orienta a la transformación de las realidades educativas del ciclo de 0-3 años (PEI, 2016). Está fundamentado en la filosofía de Reggio Emilia, que pone de manifiesto una concepción de infancia potente y capaz de construir conocimiento por sí misma. Se trabaja desde el sentido que tiene la pedagogía de la escucha que abre la ruta para el diseño de las experiencias, que es una de las fortalezas del programa; y el énfasis puesto en el ambiente como tercer educador, que invita, sugiere, inspira y provoca, como: "Múltiples formas de interacción entre las personas, los objetos y el espacio, así como nuevas formas de experimentación, descubrimiento y exploración, por parte de los niños y niñas pequeños". (Martín y Duran, 2016 pág. 42) Se tiene como referencia el modelo educativo de Loczy, pedagogía Pikler: movimiento en libertad y la teoría del vínculo de John Bowlby para proponer intervenciones pedagógicas intencionadas. 
La política pública también cobra fuerza como un eje del trabajo pedagógico. Las actividades rectoras: literatura, juego, arte y exploración del medio se hacen presentes en cada uno de los documentos. Se cita a Cabrejo, Bonnafé, Yolanda Reyes, y María Emilia. López; se hace una referencia especial al trabajo con los libros álbum, las nanas, arrullos y otras manifestaciones de la tradición oral.

El Programa hace énfasis en la pedagogía crítica para el despliegue de una praxis propia de los actores de la modalidad, potenciadora del desarrollo integral de los niños y las niñas en la primera infancia. La documentación pedagógica cumple un papel importante en este proceso de reflexión entre la teoría y la práctica.

\section{Conclusiones}

El Programa de Licenciatura en Preescolar de la Fundación Universitaria MonserrateUnimonserrate tiene importantes avances en el reconocimiento de la importancia de la práctica pedagógica desde el primer semestre con el fin de fortalecer la posibilidad de reflexión desde el propio hacer, pero también a la hora de situar prácticas en contextos comunitarios, no convencionales y formales, que implican la inmersión en diversas realidades. Las universidades no pueden estar alejadas de las realidades políticas y contextuales inmersas en los desarrollos y avances de la educación inicial; formar profesionales que lean críticamente sus realidades y contribuyan a mejorar con su acción pedagógica la calidad de vida de los niños y niñas tal como está definido en el perfil de la maestra y maestro del programa.

La escuela de educación ha estado resignificando el programa de licenciatura en Educación Preescolar con miras a dar respuesta a los avances y demandas que se han venido posicionando en materia de Educación Infantil en el país, y que aportan en la definición del perfil de los y las egresadas del programa y de la organización misma de este que, aunque siga denominándose preescolar ya ha tenido importantes reestructuraciones que responden al sentido mismo de la Educación Infantil.

La educación integral se fundamenta en un enfoque de derechos, específicamente desde la prevalencia de los derechos de niñas y niños, y el requerimiento de garantizarlos todos de manera simultánea. Hay un fuerte interés por propiciar el reconocimiento del ciclo educativo de 0-3 años y la re- significación de las prácticas pedagógicas desarrolladas en él, a partir de un ejercicio de reflexión- acción sobre la propia práctica fundamentada en el modelo de formación en documentación pedagógica de la Escuela de Educación.

El Programa ha trabajado en estudios de carácter exploratorio, en donde el objetivo es profundizar en los elementos que se deben tener en cuenta para la formación de maestras y maestros en torno a la Educación Infantil de niños y niñas entre 0 y 3 años, buscando encontrar pistas que permitan fortalecer el programa en su etapa de reestructuración, así como algunas líneas de investigación relacionadas con este campo en particular. La investigación en el programa de licenciatura en educación preescolar se sustenta en el enfoque de investigación-acción dado que se centra en la propia práctica y busca su re- significación desde la reflexión en y sobre la acción, apoyado en la incorporación del modelo de formación en documentación pedagógica de la escuela de Educación.

El Diseño de ambientes como una forma de aproximarse al trabajo pedagógico con niños y niñas menores de tres años, se retoma tanto desde la academia como desde el ámbito de la práctica. El trabajo con las familias y cuidadores es un elemento que se considera con fuerza. Este se plantea, desde el ámbito de lo práctico, sobre todo en las bebetecas y ludotecas; con fin de afianzar los lazos familiares y sociales. También, se concibe el trabajo con las familias y cuidadores, como un espacio para que se gesten los procesos de partici- 
pación en los grupos, mediante las Historias de Vida, de manera tal que se movilicen estrategias de opinión, reflexión, expresión, ratificando así la importancia de recobrar la voz de la población con la que se trabaja.

El trabajo de 0 a 3 años se señala el potenciamiento del desarrollo como una tarea pedagógica, con dos propósitos simultáneos: de un lado, la comprensión del mundo social, cultural, simbólico y contextual mediado por la comunicación humana y por el otro, potenciar conocimientos a través de los cuales se construye sentido y se viven experiencias.

Hay una apuesta fuerte por la participación infantil entendida como el camino hacia la autonomía, y como un proceso que se favorece en todo momento y lugar, desde los intereses, necesidades, ritmos y formas de participar de las niñas y los niños. Un enfoque territorial donde se considera el territorio como una construcción social, donde se viven los derechos y se constituyen relaciones que reconocen la diversidad y la diferencia o la eliminan. A este enfoque se anclan los principios de lectura de la realidad, el encuentro con lo humano y el diálogo de saberes como elementos básicos para construir.

\section{Referencias}

Espitia, L. (2017). Aportes al objeto de conocimiento en la Especialización en Educación y Orientación

Familiar de la Fundación Universitaria Monserrate. Hojas y Hablas, (14), 154-166.
DOI:10.29151/hojasyhablas.n14a12. Disponible en:http://virtual.fum.edu.co/publicaciones/ index.php/hojasyhablas/article/view/133

Fundación Universitaria Monserrate - Unimonserrate. Proyecto Educativo Institucional PEI- Proyección, cercanía y cuidado. Aprobado por El Consejo de Regencia Acuerdo No. 10 del 8 de julio 2016. Disponible en: http://www.unimonserrate.edu.co/wp-content/uploads/2017/09/PEI_Unimonserrate 2017.pdf

García y Morgan Tirado. (2007). La sistematización de experiencias: producción de conocimientos desde y para la práctica. Disponible en: http://www.ts.ucr.ac.cr/binarios/tendencias/rev-co-tendencias-15-07.pdf

Iovanovich, M. L. (2007). La sistematización de la práctica docente en educación de jóvenes y adultos. Revista Iberoamericana de Educación, 42(3). Recuperado de: https://rieoei. org/RIE/article/view/2419

Martín, M., y Durán, S. (2018). Diseño de ambientes para el juego: práctica y reflexión en educación infantil. Revista Latinoamericana de Educación Infantil, 5(1), 85-96. Recuperado de: http://www.usc.es/revistas/index.php/ reladei/article/view/4904 ISSN2255-0666.

Martín, S. (2016). Una experiencia de educación plástico visual en la sala de un año. Itinerarios Educativos, (8), 111-125. Recuperado de: https://bibliotecavirtual.unl.edu.ar/ojs/ index.php/Itinerarios/article/download/.../8968/ 